\title{
Ab Initio Studies on the Structure, Conformation, and Chain Flexibility of Halogenated Poly(thionylphosphazenes)
}

\author{
R. Jaeger, J. B. Lagowski, ${ }^{\dagger}$ I. Manners, ${ }^{*}$ and G. J. Vancso* \\ Department of Chemistry, University of Toronto, 80 St. George Street, \\ Toronto, Ontario M5S 1A1, Canada
}

Received July 28, 1994; Revised Manuscript Received October 14, $1994^{\otimes}$

\begin{abstract}
Ab initio quantum chemical calculations on short-chain model compounds have been used to study the conformation, valence electron density, and chain flexibility of halogen-substituted poly(thionylphosphazenes) (PTPs) $\left[(\mathrm{NSOX})\left(\mathrm{NPCl}_{2}\right)_{2}\right]_{n}(\mathrm{X}=\mathrm{F}$ or $\mathrm{Cl})$, which are representatives of a new class of inorganic sulfur(VI)-nitrogen-phosphorus polymers. The calculations were carried out at the closedshell Hartree-Fock level of theory using the Gaussian 92 program package. The electronic wave function was described by the 6-31G* basis set. The results show that model compounds adopt a nonplanar transcis conformation in the minimum-energy state. Based on the stable geometries of the short-chain analogues, the polymer will form a $12 / 5$ helix in its extended conformation. Rigid rotor scans and geometry optimizations of selected rotamers were used in order to investigate the torsional mobility of the main chain of the model compounds. The flexibility of the $\mathrm{S}-\mathrm{N}-\mathrm{P}$ and $\mathrm{P}-\mathrm{N}-\mathrm{P}$ bond angles contributes significantly to the chain flexibility. The torsional barriers for rotations around bonds of the PTP backbone range from 1.5 to $3.5 \mathrm{kcal} / \mathrm{mol}$. A change from chlorine to fluorine as a substituent on sulfur leads to lower torsional barriers and wider minima of the rotational potentials and therefore to an increased torsional mobility of the main chain. The increase in chain flexibility is consistent with trends in glass transition temperatures of the corresponding polymers. The electronic structure of the model compounds, including charge density distributions, is briefly discussed. The results indicate strong charge separations along the backbone of the polymer and in the direction of the substituents bonded to the main chain which are consistent with Dewar's island delocalization model.
\end{abstract}

\section{Introduction}

Research efforts targeting new polymeric materials with unusual or improved properties have traditionally focused on organic macromolecules. However, it has long been recognized that, for certain applications, carbon-based polymers are subject to a number of limitations. A possible approach to extend the range of applicability of macromolecular materials is to explore and develop the polymer chemistry based on the other 90 or so stable inorganic elements. This would increase the number of possible different combinations of the elements which form the main chain. Compared to carbon-based polymers, the novel structures will have a different skeletal geometry which can result in unusual conformations and conformational statistics. These structural differences will inevitably result in novel properties. Also, with the larger number of structural degrees of freedom, the tunability of the polymeric materials is increased. This allows us to design polymers according to specific requirements (e.g., elastomeric applications, durability in harsh environments, etc.). Even though the potential of inorganic polymers is obvious, the number of well-characterized examples of these materials is limited. Types of wellknown inorganic polymers include polysiloxanes, polysilanes, polyphosphazenes, and transition-metal-based macromolecules. ${ }^{1-3}$

Because a part of our research involves developing routes to novel classes of inorganic macromolecules, we have undertaken a systematic study of the synthesis and properties of poly(thionylphosphazenes) (PTPs)

\footnotetext{
* To whom correspondence should be addressed.

† Present address: Physics Department, Memorial University of Newfoundland, St. John's, Newfoundland A1A 3X7, Canada.

$\otimes$ Abstract published in Advance ACS Abstracts, December 1 , 1994.
}

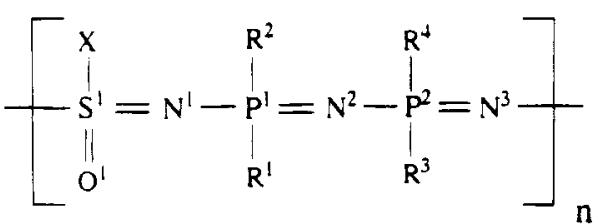

Figure 1. Repeat unit of the PTP polymer. $\mathrm{X}=\mathrm{F}, \mathrm{Cl} ; \mathrm{R}=$ $\mathrm{Cl}, p-\mathrm{OC}_{6} \mathrm{H}_{4} \mathrm{Ph}$, etc.

which possess backbones of sulfur(VI), nitrogen, and phosphorus atoms. ${ }^{4,5}$ We found that the cyclic thionylphosphazenes undergo thermally induced ring-opening polymerization and yield hydrolytically sensitive poly(thionylphosphazenes) $\left[(\mathrm{NSOX})\left(\mathrm{NPCl}_{2}\right)_{2}\right]_{n}(\mathrm{X}=\mathrm{F}$ or $\mathrm{Cl}$; see Figure 1). In reactions of these polymers with sodium aryloxides, moisture-stable poly[(aryloxy)thionylphosphazenes] [NSOCl[NP $\left.\left.\left(p-\mathrm{OC}_{6} \mathrm{H}_{4} \mathrm{Ph}\right)_{2}\right]_{2}\right]_{n}$ were obtained. Manners and co-workers determined glass transition temperatures of substituted PTPs which range from $T_{\mathrm{g}}=-56$ to $+55^{\circ} \mathrm{C} .^{5}$

Our goal is to gain an understanding of structureproperty relations of these polymers which are based on the molecular characteristics of the macromolecules. The glass transition temperature of a polymer is governed by intra- and intermolecular interactions which influence cooperative motions of chain segments. One of the factors which determines $T_{\mathrm{g}}$ is the torsional mobility of the polymer backbone: a high torsional flexibility will result in low glass transition temperatures $^{6-8}$ In contrast, polymers with a rigid main chain will exhibit high glass transition temperatures.

In this paper we report on the results of our ab initio calculations which were used to study the structure and chain flexibility of halogen-substituted PTPs. We use small molecular weight analogues of PTPs which consist of one repeat unit of the polymer in order to carry out the computations. These analogue structures-often 
called "mimics"-are used to simulate the conformational energy map of the polymer. First we will discuss the ground-state geometries of model compounds with fluorine or chlorine bonded to sulfur and chlorine bonded to phosphorus. Then we will determine the torsional potentials of selected bonds of the main chain. The torsional potentials describe how much energy is required to rotate a part of the molecule around a bond and, therefore, are a quantitative measure of the torsional flexibility of the polymer.

\section{Ab Initio Molecular Orbital Calculations of Molecular Geometries and Torsional Potentials}

$\mathrm{Ab}$ initio quantum mechanical calculations solve Schrödinger's equation of the molecular electronic wave function for a given molecular geometry. ${ }^{9}$ A basic result of the ab initio calculations is the energy of a molecular geometry with respect to the quantum chemical standard state (i.e., the state where all electrons and nuclei of the compound are infinitely separated). The energy of a molecule as a function of its internal coordinates (i.e., all bond lengths and bond and dihedral angles which are necessary in order to define its geometry) is commonly referred to as the potential energy surface of the molecule. The ab initio calculations determine points of the potential energy surface. The stable conformations of a molecule correspond to minima of the potential energy surface. Therefore, minimizing the energy of a molecule as a function of its internal coordinates will determine its stable conformations. The procedure of searching for the stable conformations is called geometry optimization. The energy which is needed to rotate parts of a molecule around a given bond is-at least in theory-easily obtained. As a first step, the stable conformation of the molecule is determined. Starting from the stable conformation, the energies of the conformers which form during a given rotation are calculated. These energies represent the torsional potential of the rotation. Therefore, ab initio calculations provide a useful way to map the torsional potential of rotations around specific bonds. At this point, we want to emphasize a characteristic of the ab initio method: Interactions between the molecule and, e.g., solvent molecules or neighboring molecules are usually not included in the calculations. Therefore, the results of ab initio calculations only apply for an individual molecule in the gas phase.

The drastic increase in computer time which is needed for calculations on "larger" compounds is the major limitation of the applicability of the ab initio method in polymer science: the computer time required increases approximately with the fourth power of the number of atomic orbitals which are used to carry out the computations. ${ }^{10}$ In order to overcome this problem, the possibility of using less costly semiempirical or classical force field (molecular mechanics) calculations has been evaluated. Unfortunately, these methods rely on a set of parameters for the compound to be studied which can only be obtained from experimental data. Since suitable parametrizations for PTPs are not yet available, semiempirical and classical force field calculations were ruled out for our study.

In order to avoid lengthy computations, we chose a model system which represents the geometry and flexibility of the polymer backbone in an adequate way and allowed us to carry out the ab initio calculations in a reasonable period of time. We performed the calculations on two low molecular weight analogues. The<smiles>[X][Si](C)(=O)=NP(Cl)(Cl)=NP(Cl)(Cl)=NC</smiles>

Figure 2. Chemical structure of "mimic 1". $\mathrm{X}=\mathrm{F}, \mathrm{Cl}$.<smiles>[X]S(=O)(N=[P+](Cl)(Cl)Cl)=NN=P(C)(Cl)Cl</smiles>

Figure 3. Chemical structure of "mimic 2". $\mathrm{X}=\mathrm{F}, \mathrm{Cl}$. model compounds or mimics consisted of one polymer repeat unit which was completed on both ends with a methyl group (see Figures 2 and 3 ). In another part of our study we rearranged the repeat unit in order to assess the effect of the methyl end groups on the molecular geometry.

We used two approaches to study the torsional potentials. In order to gain a first impression of the torsional flexibility of the polymer backbone, we performed a series of "rigid rotor scans". In the "rigid rotor" approximation all bond lengths, bond angles, and remaining dihedral angles are kept fixed while a part of the molecule is rotated around a bond. This means that the rigid rotor approximation yields an upper limit for the actual torsional potential.

In addition, we compared the results of the rigid rotor scans with results of "fully optimized" or "relaxed" scans around selected bonds. In the relaxed scans the energies of fully optimized structures which form during a rotation are determined. Therefore, the relaxed scans will yield torsional barrier shapes of the torsional potentials which are close to the ones in reality.

The rigid rotor scans are-compared to the relaxed scans-significantly less time-consuming, since geometry optimizations are omitted while one part of the molecule is rotated around a bond. Therefore, the rigid rotor approximation is not successful in determining absolute barrier heights and shapes of torsional potentials. However, the rigid rotor model is capable of reproducing changes in torsional potentials caused by different substituents. ${ }^{9}$

We chose model compounds with chlorine substituents on the phosphorus atoms of the backbone and fluorine or chlorine substituents on sulfur atoms of the backbone. The chemical synthesis and some characteristics of these compounds have been reported earlier. ${ }^{5}$ These results showed that if $\mathrm{Cl}$ instead of $\mathrm{F}$ is bonded to sulfur, the glass transition temperature of the polymer increases by $10{ }^{\circ} \mathrm{C}$ from -56 to $-46{ }^{\circ} \mathrm{C}$. Fluorine and chlorine are "small" substituents compared with, e.g., aryloxy groups, and so the calculations can be completed in a reasonable period of time. Moreover, single atoms are cylindrically symmetric substituents. Therefore, we do not neglect a torsional degree of freedom if we apply the rigid rotor approximation. The major shortcoming of the rigid rotor approximation is the neglect of the flexibility of the $\mathrm{S}-\mathrm{N}-\mathrm{P}$ and $\mathrm{P}-\mathrm{N}-\mathrm{P}$ bond angles. A comparison of the results of rigid rotor scans and energies of geometry-optimized rotamers (relaxed scans) will show the contribution of the flexibility of the S-N-P and $\mathrm{P}-\mathrm{N}-\mathrm{P}$ bond angles to the torsional mobility of the PTP backbone.

\section{Method of Computation}

We used a HP 755 workstation and a Indigo SGI RS 4000 workstation in order to perform the Gaussian 92 
ab initio calculations. ${ }^{11,12}$ The calculations were carried out at the closed-shell Hartree-Fock SCF level of theory, using the $6-31 \mathrm{G}^{*}$ polarized split-valence basis set for the gaussian orbital wave functions. The program employs a gradient method for geometry optimizations.

Polarized split-valence basis sets include additional atomic orbital functions in order to enhance the capability of the algorithm to model the molecular orbitals. Minimal basis sets only consist of the number of atomic orbital functions which is required to accomodate all of the electrons of the atoms. Split-valence basis sets include two sets of valence orbitals which enable the algorithm to expand and contract the wave functions in response to differing molecular environments. A polarized basis set incorporates functions of higher angular momentum number than are needed by the atom in its electronic ground state (p-type orbital functions for hydrogen and helium, d-type orbital functions for second and third row elements). The "polarization functions" permit displacements of the center of the electronic charge away from the nucleus of the atom.

We determined the influence of electron correlation for two conformations, using single-point 2nd-order Møller-Plesset perturbation theory calculations.

The graphical representations of the model compounds were created with the CERIUS molecular modeling software package. ${ }^{13}$

\section{Stable Geometries of the Model Compounds}

The stable geometry of short-chain analogues of PTPs with several different substituents has been the subject of our previous studies. ${ }^{14,15}$ The results for chlorine as a substituent on phosphorus and chlorine or fluorine as a substituent on sulfur are reviewed in this paper. In our previous studies and in the discussion which is presented below we conclude that the model compounds adopt a nonplanar trans-cis conformation (see Figures

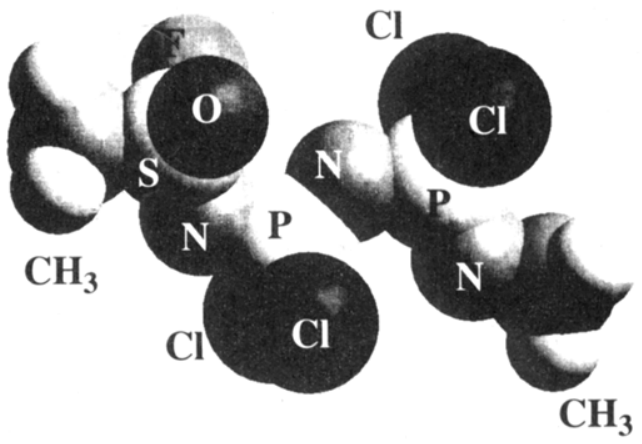

Figure 4. Trans-cis conformations of mimic 1 with $\mathrm{F}$ as a substituent bonded to sulfur.

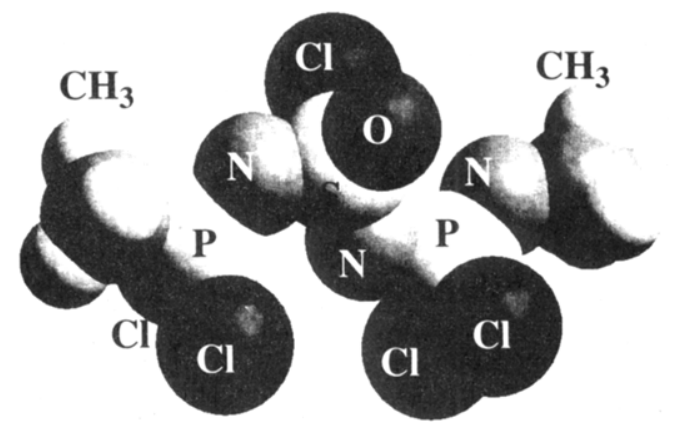

Figure 5. Trans-cis conformations of mimic 2 with $\mathrm{Cl}$ as a substituent bonded to sulfur.

4 and 5 ). The values of the bond lengths, bond angles, and dihedral angles in the stable conformation are shown in Table 1 . In order to assess the accuracy of the calculated bond lengths, bond angles, and dihedral angles, we carried out a RHF/6-31G* geometry optimization and an X-ray diffraction study on a bis(phosphazo) sulfone. ${ }^{16}$ The results showed that the ab initio calculation reproduced the measured bond lengths of the main chain (i.e., $\mathrm{S}-\mathrm{N}$ and $\mathrm{P}=\mathrm{N}$ bond lengths) with an accuracy of $\pm 0.009 \AA$. The bond lengths for substituents

Table 1. Bond Lengths, Bond Angles, and Dihedral Angles of the Model Compounds

\begin{tabular}{|c|c|c|c|c|c|c|}
\hline & \multicolumn{2}{|c|}{ mimic 1 trans-cis } & \multicolumn{2}{|c|}{ mimic 2 trans - cis } & \multicolumn{2}{|c|}{ mimic 2 cis-trans } \\
\hline & $\mathbf{X}=\mathbf{F}$ & $\mathrm{X}=\mathrm{Cl}$ & $\mathbf{X}=\mathbf{F}$ & $\mathrm{X}=\mathrm{Cl}$ & $\mathbf{X}=\mathbf{F}$ & $\mathrm{X}=\mathrm{Cl}$ \\
\hline \multicolumn{7}{|c|}{ Bond Lengths (Å) } \\
\hline $\mathrm{N}^{3}-\mathrm{S}^{1}$ & & & 1.572 & 1.580 & 1.570 & 1.577 \\
\hline $\mathrm{S}^{1}=\mathrm{N}^{1}$ & 1.503 & 1.515 & 1.513 & 1.522 & 1.483 & 1.492 \\
\hline $\mathrm{N}^{1}-\mathrm{P}^{1}$ & 1.628 & 1.627 & 1.631 & 1.632 & 1.632 & 1.631 \\
\hline $\mathrm{P}^{1}=\mathrm{N}^{2}$ & 1.531 & 1.531 & 1.500 & 1.499 & 1.495 & 1.494 \\
\hline $\mathrm{N}^{2}-\mathrm{P}^{2}$ & 1.608 & 1.607 & & & & \\
\hline $\mathrm{P}^{2}=\mathrm{N}^{3}$ & 1.509 & 1.509 & 1.554 & 1.556 & 1.556 & 1.559 \\
\hline $\mathrm{O}=\mathrm{S}^{1}$ & 1.414 & 1.423 & 1.409 & 1.417 & 1.421 & 1.429 \\
\hline $\mathrm{X}-\mathrm{S}^{1}$ & 1.558 & 2.022 & 1.556 & 2.026 & 1.559 & 2.026 \\
\hline $\mathrm{P}-\mathrm{Cl}$ & $2.025^{a}$ & $2.026^{a}$ & $2.023^{a}$ & $2.023^{a}$ & $2.018^{a}$ & $2.023^{a}$ \\
\hline \multicolumn{7}{|c|}{ Bond Angles (deg) } \\
\hline$\angle \mathrm{P}^{2} \mathrm{~N}^{3} \mathrm{~S}^{1}$ & & & 130.8 & 130.4 & 128.9 & 128.1 \\
\hline$\angle \mathrm{N}^{3} \mathrm{~S}^{1} \mathrm{~N}^{1}$ & & & 107.4 & 106.4 & 112.9 & 112.0 \\
\hline$\angle \mathrm{O}^{1} \mathrm{~S}^{1} \mathrm{~N}^{1}$ & 122.3 & 121.4 & 122.0 & 121.0 & 116.9 & 116.1 \\
\hline$\angle \mathrm{S}^{1} \mathrm{~N}^{1} \mathrm{P}^{1}$ & 128.9 & 128.4 & 126.9 & 127.0 & 139.8 & 139.7 \\
\hline$\angle \mathrm{N}^{1} \mathrm{P}^{1} \mathrm{~N}^{2}$ & 112.8 & 112.8 & 115.5 & 115.5 & 118.8 & 118.8 \\
\hline$\angle \mathrm{P}^{1} \mathrm{~N}^{2} \mathrm{P}^{2}$ & 133.5 & 133.9 & & & & \\
\hline$\angle \mathrm{N}^{2} \mathrm{P}^{2} \mathrm{~N}^{3}$ & 115.1 & 115.2 & & & & \\
\hline \multicolumn{7}{|c|}{ Dihedral Angles (deg) } \\
\hline$\angle \mathrm{C}^{1} \mathrm{P}^{2}-\mathrm{N}^{3} \mathrm{~S}^{1}$ & & & 184 & 182 & -53 & -52 \\
\hline$\angle \mathrm{P}^{2} \mathrm{~N}^{3}-\mathrm{S}^{1} \mathrm{~N}^{1}$ & & & -12 & -9 & 145 & 138 \\
\hline$\angle \mathrm{N}^{3} \mathrm{~S}^{1}-\mathrm{N}^{2} \mathrm{P}^{1}$ & & & 182 & 186 & 27 & 19 \\
\hline$\angle \mathrm{O}^{1} \mathrm{~S}^{1}-\mathrm{N}^{1} \mathrm{P}^{1}$ & -49 & -50 & -47 & -45 & 161 & 150 \\
\hline$\angle \mathrm{S}^{1} \mathrm{~N}^{1}-\mathrm{P}^{1} \mathrm{~N}^{2}$ & -28 & -30 & -29 & -32 & 163 & 167 \\
\hline$\angle \mathrm{N}^{1} \mathrm{P}^{1}-\mathrm{N}^{2} \mathrm{P}^{2}$ & 181 & 182 & & & & \\
\hline$\angle \mathrm{P}^{1} \mathrm{~N}^{2}-\mathrm{P}^{2} \mathrm{~N}^{3}$ & 1 & 2 & & & & \\
\hline$\angle \mathrm{N}^{2} \mathrm{P}^{2}-\mathrm{N}^{3} \mathrm{C}^{2}$ & 180 & 180 & & & & \\
\hline
\end{tabular}

${ }^{a}$ An average value of the corresponding quantities. A dihedral angle $\phi=0$ corresponds to the cis conformation. 
bonded to the main chain differed by a maximum of $\pm 0.031 \AA$. The maximum difference between measured and calculated bond angles was $3.5^{\circ}$. The calculations did not systematically over- or underestimate bond lengths and bond angles. We observed significant differences in dihedral angles resulting from calculations and dihedral angles obtained from the X-ray diffraction study. This result is consistent with our findings concerning the rotational mobility of the main chain, which will be discussed in the subsequent sections. The calculated geometry represents a molecule in the gas phase. The conformation of a molecule in the crystalline phase is (in particular for highly flexible molecules) strongly influenced by strains which are imposed on the molecule by crystal packing.

Geometry of the Ground-State Trans-Cis Conformation. The stable conformation exhibits a pattern of slightly alternating bond lengths along the main chain. The longer, "single" bonds lie parallel to the direction of the main chain, and the shorter "double" bonds are almost perpendicular to the direction of the main chain. The average difference between single and double phosphorus-nitrogen bonds is $0.11 \AA$. This result is in contrast to X-ray studies on substituted linear phosphazene oligomers and poly(dichlorophosphazene) (PDCP) which were performed by Allcock and co-workers. ${ }^{17-19}$ They considered bond length alternations of $0.05 \AA$ for the phosphazene oligomers as insignificant and suggested several models for the structure of PDCP with no $\mathrm{P}-\mathrm{N}$ bond length alternations. Two questions arise from these contradicting observations: Are the bond length alternations which are observed in the ab initio results real? If the bond length alternations observed for short-chain molecules of PTPs exist, what are the consequences for the corresponding polymer?

Roesky and co-workers determined the structure of a silylated derivative of the phosphazenium salt $\left[\mathrm{H}_{2} \mathrm{~N}\right.$ $\left.\mathrm{PPh}_{2} \mathrm{NPPh}_{2} \mathrm{NH}_{2}\right]^{+} \mathrm{Cl}^{-}$using $\mathrm{X}$-ray diffraction and observed bond length alternations in the range of $0.06-$ $0.12 \AA .^{20}$ An ab initio study on short-chain model compounds of poly(methyloxothiazene) $\left([\mathrm{N}=\mathrm{S}(\mathrm{O}) \mathrm{Me}]_{n}\right)$ carried out by Roy et al. showed slightly alternating bond lengths between sulfur-nitrogen bonds. ${ }^{21}$ Another X-ray diffraction study on PDCP carried out by Chatani and Yatsuyanagi suggests a trans - cis conformation with glide symmetry and significant $\mathrm{P}-\mathrm{N}$ bond length alternations. ${ }^{22}$ Ferris and Duke observed bond length alternations in an ab initio study of short-chain analogues of polyphosphazenes. ${ }^{23}$ They suggested-with regard to the structure of polyphosphazenes proposed by Allcock-that the end groups cause the alternation of $\mathrm{P}-\mathrm{N}$ bond lengths along the main chain. Since the influence of the end groups is more prominent in short linear species than in the polymer, they concluded that the bond length alternations are a characteristic of low molecular weight molecules. Although chemical bonding in linear phosphazene, oxothiazene, and thionylphosphazene backbones cannot be interpreted in terms of single and double bonds, we believe that the slight alternations of bond lengths observed in small, linear molecules of substituted thionylphosphazenes are real. Unlike in cyclic small molecules of phosphazenes or thionylphosphazenes, the trans-cis conformation of a linear molecule possesses no symmetry which forces a molecular geometry with equal $\mathrm{P}-\mathrm{N}$ or $\mathrm{S}-\mathrm{N}$ bond lengths to be a stationary point of the potential energy surface. On the basis of this conclusion, one might ask

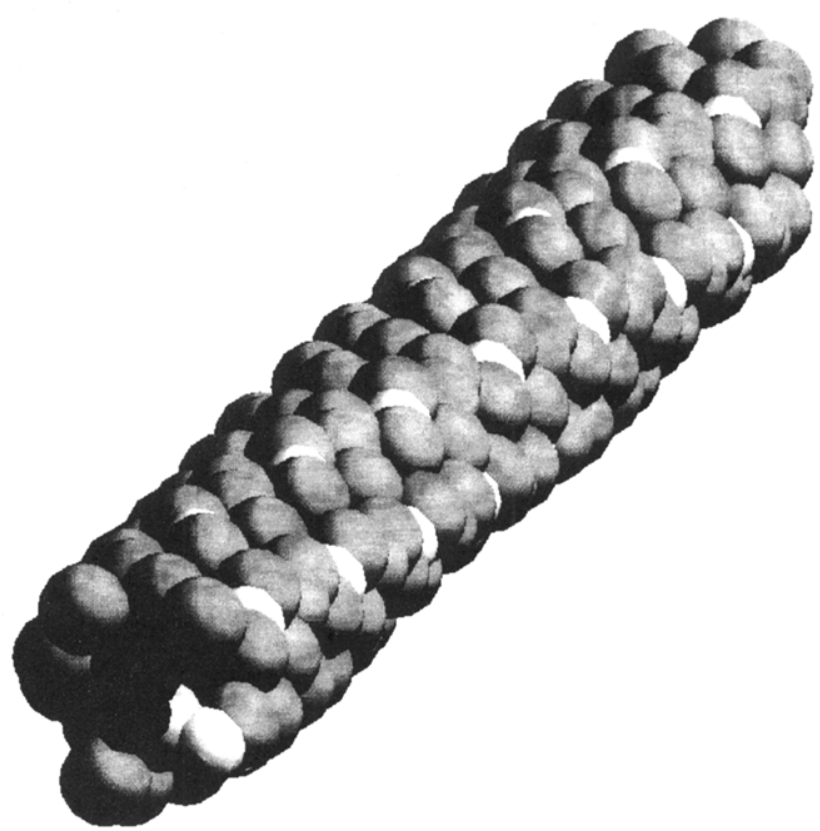

Figure 6. Helical structure of the PTP polymer with chlorine as a substituent on sulfur and phosphorus in its extended conformation. The oxygen atoms on sulfur are colored white in order to make the helix pitch visible.

whether cyclic molecules represent better model systems for the bond structure of the polymer since they are-in contrast to short-chain linear molecules-not influenced by end-group effects. Longuet-Higgins and Salem investigated alternating bond lengths in cyclic polyenes $\left(\mathrm{C}_{4 n+2} \mathrm{H}_{4 n+2}\right)$ and concluded that a configuration of equal bond lengths becomes unstable with respect to a conformation with alternating bond lengths in the limit $n$ $\rightarrow \infty .{ }^{24}$ Considering Ferris and Duke's as well as Longuet-Higgins and Salem's conclusions on bond length alternations, we can state that both linear and cyclic small molecules have certain shortcomings as model systems for the corresponding polymer. However, for our purpose we have to rule out cyclic molecules, since full rotations of a part of a molecule around a bond of the main chain can only be performed with linear molecules.

The two different substituents on sulfur cause the molecule to deviate from a planar trans-cis conformation. Due to the nonplanarity of the PTP monomer unit, the PTP polymer will form a helix in its stretched conformation. We used the molecular geometries of mimic 1 and mimic 2 in order to determine the helix type. The polymer chain forms to a good approximation a $12 / 5$ helix with a 86 - $\AA$-long repeat unit which consists of 12 monomers (see Figure 6).

A Local Minimum of the Potential Energy Surface: The Cis-Trans Conformer. We used the stable conformations of the model compounds as starting points for rigid rotor and relaxed scans. If the transcis conformation is inspected, it becomes obvious that this structure is well suited for scans around the single bonds which lie parallel to the direction of the main chain. For a full rotation around a double bond, extensive rearrangements of the conformation of the molecules are necessary in order to avoid that one end of the molecule interferes with the other end. Since the rigid rotor model does not allow any relaxation of the molecular geometry during the rotations, the rigid rotor approximation would fail to give reasonable results for a full rotation around double bonds. The relaxations of the molecular geometry for a fully optimized scan 


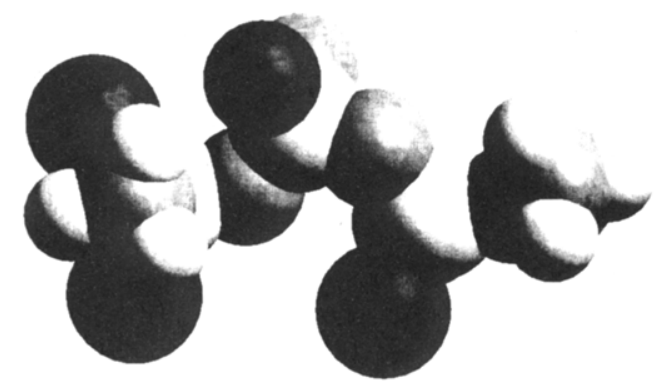

Figure 7. Cis-trans conformations of mimic 2 with $\mathrm{F}$ as a substituent bonded to sulfur.

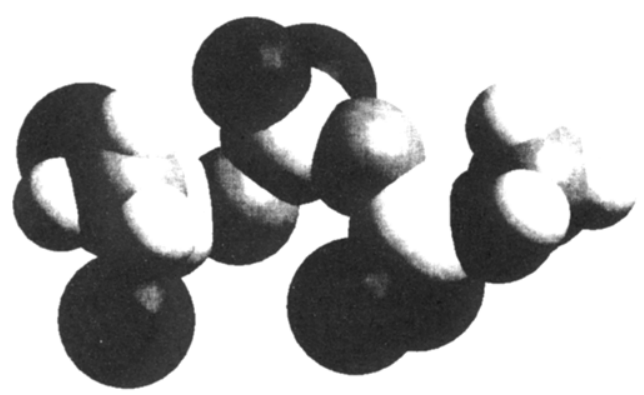

Figure 8. Cis-trans conformations of mimic 2 with $\mathrm{Cl}$ as a substituent bonded to sulfur.

around one of the double bonds would require timeconsuming geometry optimizations. This problem led us to ask the question of whether another stable conformation of the molecules exists-one in which the double bonds lie parallel and the single bonds lie almost perpendicular to the direction of the main chain. A change of every dihedral which describes the orientation of the main chain by $180^{\circ}$ generates the starting point for a geometry optimization for the cis-trans conformation. The geometrical parameters for the optimized cistrans conformations of mimic 2 are also shown in Table 1. The cis-trans conformers correspond to a local minimum of the potential energy surface which lies 6.87 $(\mathrm{X}=\mathrm{F})$ or $7.05 \mathrm{kcal} / \mathrm{mol}(\mathrm{X}=\mathrm{Cl})$ higher than the absolute minimum. Thus, the double bonds cannot be parallel to the direction of the main chain in the ground state.

If we compare the $\mathrm{S}^{1} \mathrm{~N}^{1} \mathrm{P}^{1}$ bond angles of the transcis conformers $\left(\angle \mathrm{S}^{1} \mathrm{~N}^{1} \mathrm{P}^{1}=126.9^{\circ}\right)$ with the corresponding bond angles for the cis-trans conformers $\left(\angle \mathrm{S}^{1} \mathrm{~N}^{1} \mathrm{P}^{1}\right.$ $=139.8^{\circ}$ ), we observe that these angles show an unusual flexibility compared to $\mathrm{N}-\mathrm{P}-\mathrm{N}$ or $\mathrm{N}-\mathrm{S}-\mathrm{N}$ bond angles. Earlier studies on linear short-chain or cyclic species of substituted phosphazenes ${ }^{17,18}$ and short-chain analogues of substituted PTPs ${ }^{14,15}$ already reported on the flexibility of $\mathrm{P}-\mathrm{N}-\mathrm{P}$ and $\mathrm{S}-\mathrm{N}-\mathrm{P}$ bond angles: the corresponding values depend strongly on the substituents which are bonded to the main chain as well as on whether species with a linear or cyclic backbone are investigated.

The methyl end groups of the model compounds in the cis - trans conformation are not in the plane defined by the $\mathrm{S}^{1}, \mathrm{~N}^{3}$, and $\mathrm{P}^{2}$ or the $\mathrm{N}^{1}, \mathrm{P}^{1}$, and $\mathrm{N}^{2}$ atoms (see Figures 7 and 8 ). This is in contrast to the results obtained for the trans-cis conformation.

Valence Electron Density of the Model Compounds. The charge density of the valence electrons of mimic 2 with fluorine as a substituent on sulfur is displayed in Figure 9. The valence electron density exhibits nodes at both phosphorus and sulfur and a buildup of electron density at nitrogen. This charge distribution is in agreement with the classical picture

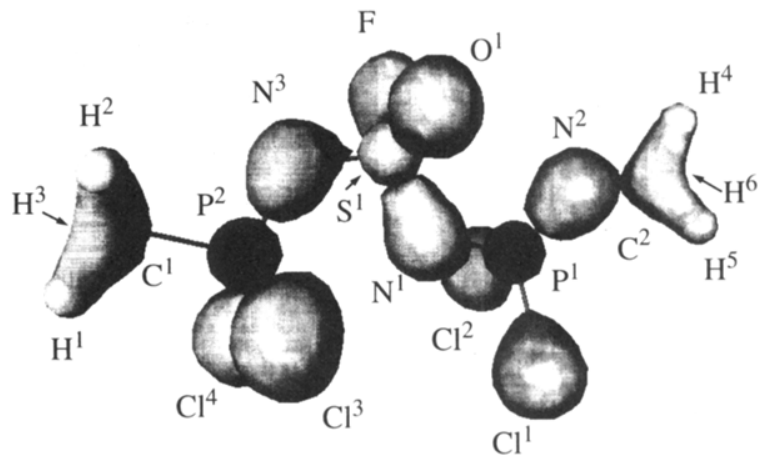

Figure 9. Charge density of the valence electrons of mimic 2 with fluorine as a substituent on sulfur. The surface denotes the volume which includes $85 \%$ of the charge density of the valence electrons.

Table 2. "Mimics" Which Were Chosen for Particular Bond Rotations

\begin{tabular}{cc}
\hline dihedral angle & model compound \\
\hline$\angle \mathrm{Cl}^{3} \mathrm{P}^{2}=\mathrm{N}^{3} \mathrm{~S}^{1}$ & mimic 2, cis-trans conformation \\
$\angle \mathrm{P}^{2} \mathrm{~N}^{3}-\mathrm{S}^{1} \mathrm{~N}^{1}$ & mimic 2, trans-cis conformation \\
$\angle \mathrm{N}^{3} \mathrm{~S}^{1}=\mathrm{N}^{1} \mathrm{P}^{1}$ & mimic 2, cis-trans conformation \\
$\angle \mathrm{S}^{1} \mathrm{~N}^{1}-\mathrm{P}^{1} \mathrm{~N}^{2}$ & mimic 2, trans-cis conformation \\
$\angle \mathrm{P}^{1} \mathrm{~N}^{2}-\mathrm{P}^{2} \mathrm{~N}^{3}$ & mimic 1, trans-cis conformation
\end{tabular}

of bonding in linear and cyclic phosphazenes involving the island model elucidated by Dewar. ${ }^{25}$ This model states that a delocalization of valence electrons occurs over only three atom $\mathrm{P}-\mathrm{N}-\mathrm{P}$ units. The charge density adopts an "islandlike" structure with nodes on phosphorus and high charge densities around nitrogen. The calculations show that the molecule possesses a highly polar backbone. Various population analysis schemes like the Mulliken population analysis ${ }^{26}$ and the natural population analysis ${ }^{27}$ assign strong partial charges to the skeletal atoms. We therefore anticipate that electrostatic repulsion or attraction of atoms will give a significant contribution to the intra- and intermolecular interactions. A study of the solution properties of poly[(aryloxy)thionylphosphazene] [NSOCl[NP $\left(p-\mathrm{OC}_{6} \mathrm{H}_{4}\right.$ $\left.\left.\mathrm{Ph})_{2}\right]_{2}\right]_{n}$ by dynamic light scattering and viscometry showed that a small amount of the quaternary ammonium salt $\left[\mathrm{Bu}_{4} \mathrm{~N}\right] \mathrm{Br}$ reduces the effective hydrodynamic radius of the macromolecules in dilute solutions. The shrinking of the radius of the coil was interpreted in terms of an interaction of the partial electronic charges of the polymer skeleton and the salt. ${ }^{28}$

\section{Rotational Flexibility of the PTP Backbone}

We investigated the torsional flexibility of five bonds of the PTP backbone using the rigid rotor approximation. All scans were carried out for fluorine as well as for chlorine as a substituent on sulfur. For three bonds we determined optimized geometries and energies of selected rotamers which form during the torsions. In order to minimize the influence of the methyl end groups on the torsional potentials, we chose the mimics for the scans where the rotated bond was situated close to the center of the molecule. Table 2 summarizes the mimics which were chosen for particular bond rotations.

Rigid Rotor Scans. We performed the rigid rotor scans in $30^{\circ}$ steps, starting from the stable conformation of the model compounds. The results of the rigid rotor scans are displayed in Figures 10 and 11. A comparison of the results for different substituents on sulfur shows that the substituents influence the rotational flexibility of the main chain. The change of chlorine to fluorine as a substituent on sulfur leads to lower torsional 

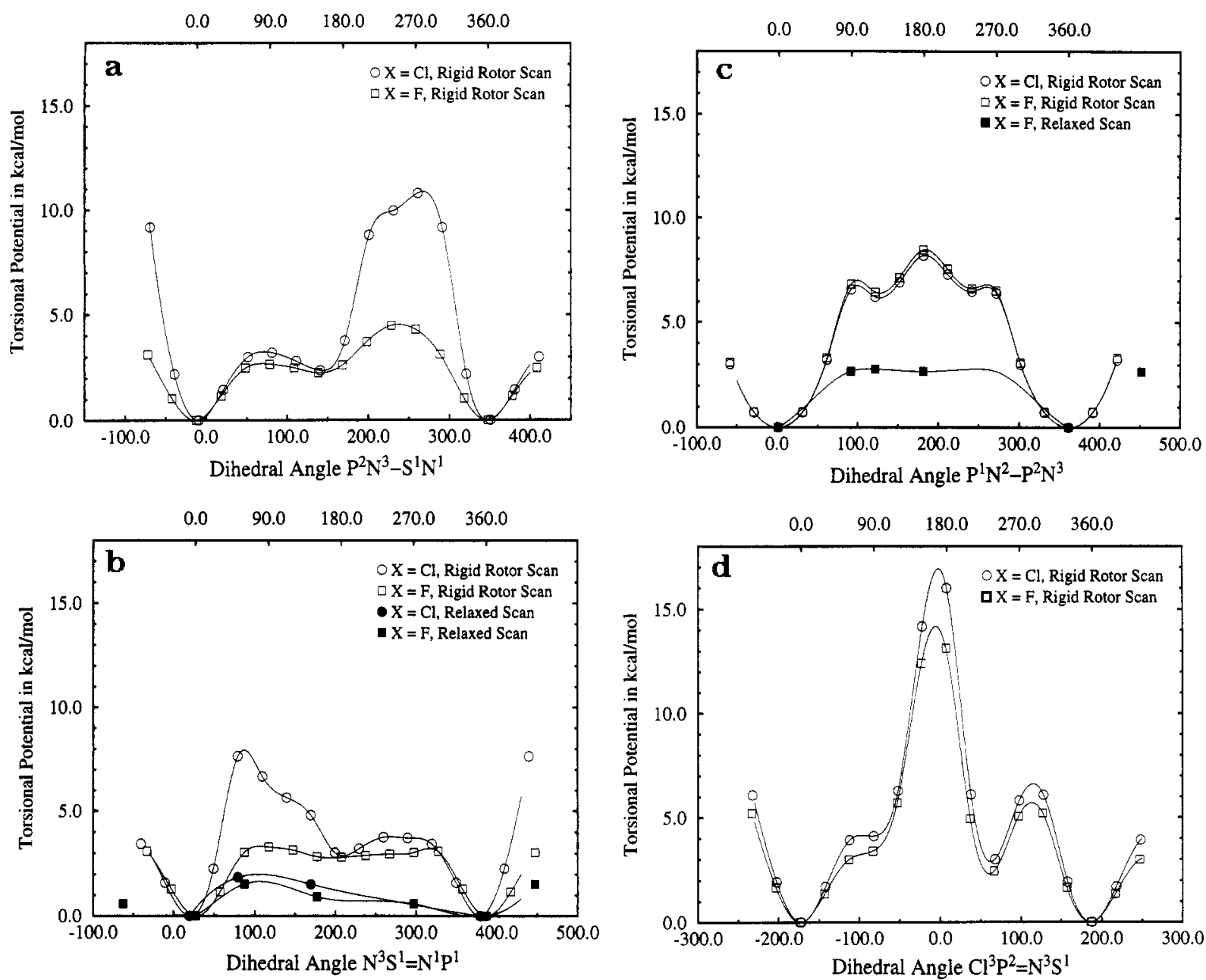

Figure 10. Torsional potentials of the $N^{3}-S^{1}(a), S^{1}=N^{1}(b), N^{2}-P^{2}(c)$, and $P^{2}=N^{3}$ (d) bonds. Open symbols denote results of rigid rotor scans; filled symbols denote results of relaxed scans. The top $x$-scale shows the angle by which one part of the molecule was rotated, starting from the stable geometry. The bottom $x$-scale displays the absolute torsional angle where $\phi=0$ corresponds to the cis conformation.

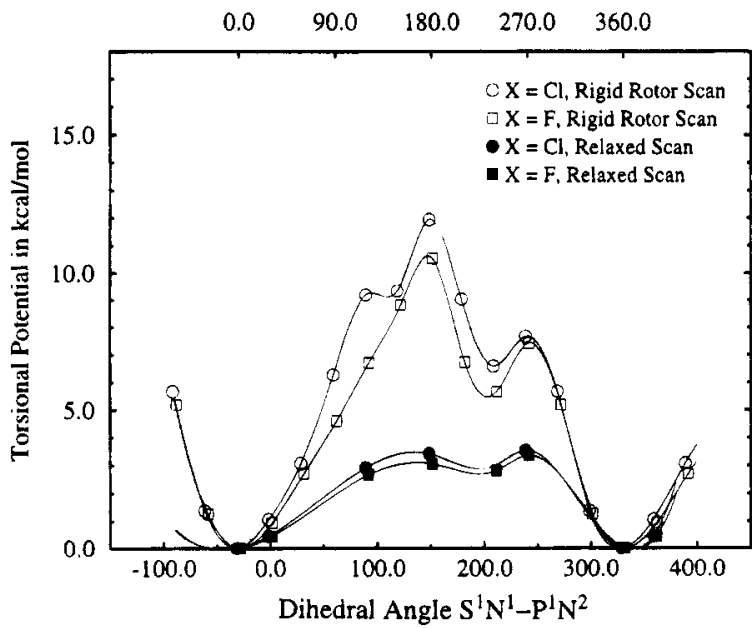

Figure 11. Torsional potential of the $\mathrm{N}^{1}-\mathrm{P}^{1}$ bond. Open symbols denote results of rigid rotor scans; filled symbols denote results of relaxed scans. The top $x$-scale shows the angle by which one part of the molecule was rotated, starting from the stable geometry. The bottom $x$-scale displays the absolute torsional angle where $\phi=0$ corresponds to the cis conformation.

barriers and wider global minima for four of the potentials. Only the flexibility of the $\mathrm{N}^{2}-\mathrm{P}^{2}$ bond of the "phosphazene" end of mimic 1 (potential c in Figure 10) is not affected by the change of the substituent on sulfur. This observation justifies the use of low molecular weight mimics in order to simulate the chain flexibility of the PTP polymer: the torsional mobility of a bond is governed by its local molecular environment which is the substituents closest to the selected bond of the main chain.

The shape of the torsional potentials which were obtained with the "rigid rotor" approximation can be explained by nonbonded interactions of the substituents bonded to the main chain. We chose the rotational potential which is displayed in Figure 11 as an example: The rotational potential of the $\mathrm{N}^{1}-\mathrm{P}^{1}$ bond exhibits three maxima for chlorine as a substituent on sulfur. The first maximum occurs at the dihedral angle $\angle \mathrm{S}^{1} \mathrm{~N}^{1}-\mathrm{P}^{1} \mathrm{~N}^{2}=90^{\circ}$ (see Figure $12 \mathrm{~b}$ ) which minimizes the distance between chlorine on sulfur and substituent $\mathrm{Cl}^{2}$ on phosphorus. A further rotation around the $\mathrm{N}^{1}-$ $P^{1}$ bond leads to the global maximum of the torsional potential which corresponds to the eclipsed position of substituents on sulfur and substituents on phosphorus $\left(\angle \mathrm{S}^{1} \mathrm{~N}^{1}-\mathrm{P}^{1} \mathrm{~N}^{2}=150^{\circ}\right.$; see Figure $\left.12 \mathrm{c}\right)$. The conformation for which the distance between oxygen on sulfur and $\mathrm{Cl}^{1}$ on phosphorus becomes minimal can be assigned to the third maximum $\left(\angle \mathrm{S}^{1} \mathrm{~N}^{1}-\mathrm{P}^{1} \mathrm{~N}^{2}=240^{\circ}\right.$; see Figure 12d). If chlorine is replaced by fluorine on sulfur, the first maximum disappears. We were able to qualitatively reproduce the results of the rigid rotor scans by calculating the electrostatic interaction between the partial atomic charges determined with the Mulliken or natural population analysis. The barrier heights 


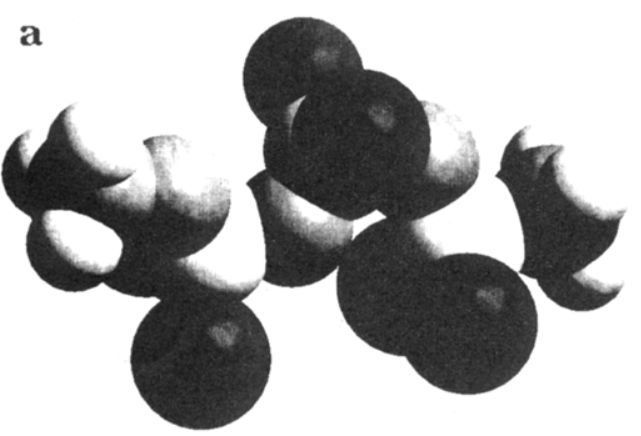

C

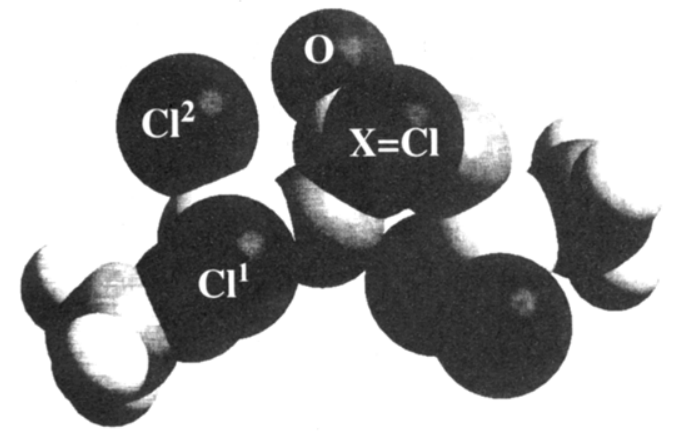

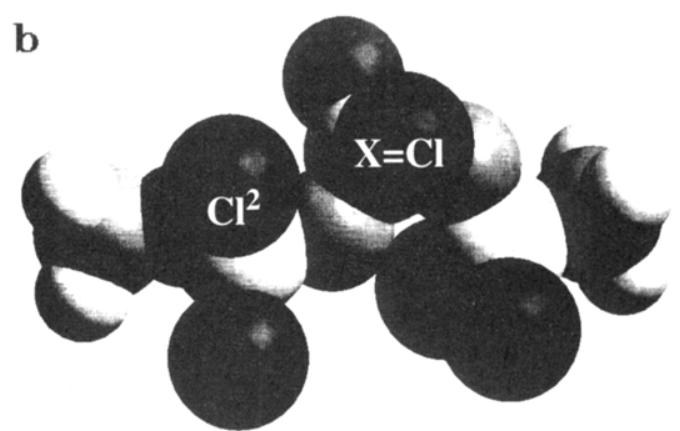

d

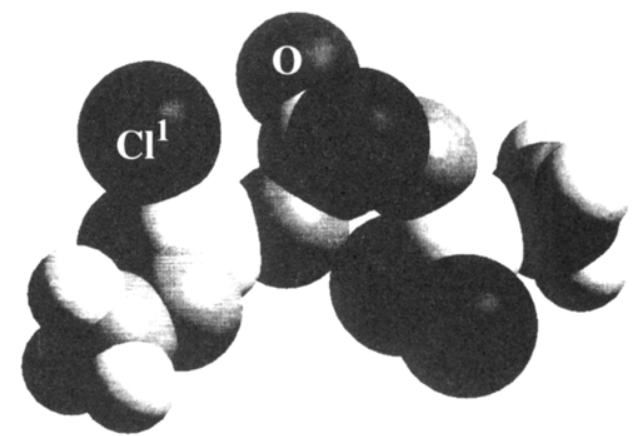

Figure 12. Ground-state conformation and three rotamers of mimic 2. The rotamers correspond to relative and absolute maxima of the rotational potential which was obtained with the rigid rotor approximation.

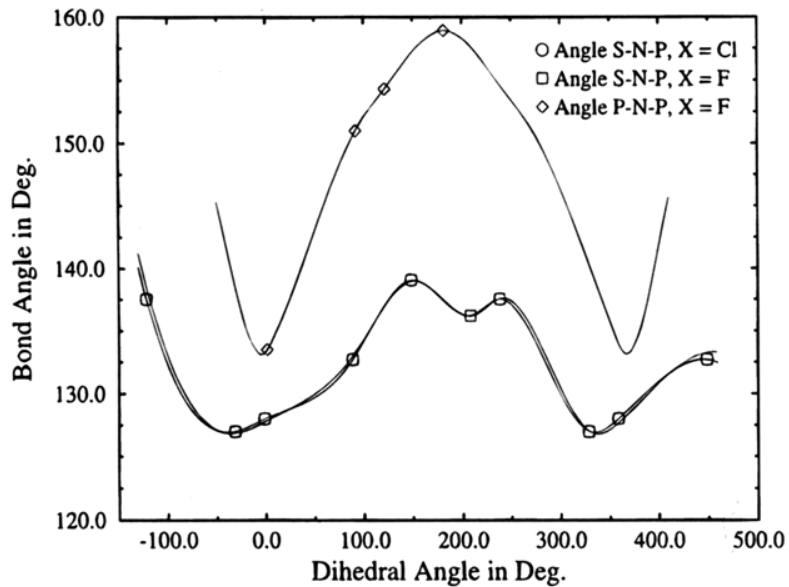

Figure 13. Change in the $\mathrm{S}^{1}-\mathrm{N}^{1}-\mathrm{P}^{1}$ bond angle during a torsional motion around the $\mathrm{N}^{1}-\mathrm{P}^{1}$ bond and the change in the $\mathrm{P}^{1}-\mathrm{N}^{2}-\mathrm{P}^{2}$ bond angle during a torsion of the $\mathrm{N}^{2}-\mathrm{P}^{2}$ bond.

obtained with the rigid rotor approximation (which range from 3.5 to $13 \mathrm{kcal} / \mathrm{mol}$ for fluorine as a substituent on sulfur and range from 7.5 to $16 \mathrm{kcal} / \mathrm{mol}$ for chlorine as a substituent on sulfur) provide an upper limit for the actual torsional barriers. Furthermore, the shape of the preceding torsional potentials will change if the molecular geometry is relaxed during a rotation around a bond.

Relaxed Scans. We carried out geometry optimizations of rotamers which form during torsions around the $\mathrm{N}^{1}-\mathrm{P}^{1}$ bond (see Figure 11), the $\mathrm{S}^{1}=\mathrm{N}^{1}$ bond (see Figure $10 \mathrm{~b}$ ), and the $\mathrm{N}^{2}-\mathrm{P}^{2}$ bond (see Figure 10c). The $\mathrm{S}-\mathrm{N}-\mathrm{P}$ and $\mathrm{P}-\mathrm{N}-\mathrm{P}$ bond angles change significantly during a full rotation of a part of the molecule. The $\mathrm{S}^{1}-\mathrm{N}^{1}-\mathrm{P}^{1}$ bond angle opens by approximately $10^{\circ}$ for both $\mathrm{F}$ and $\mathrm{Cl}$ as a substituent on $\mathrm{S}$ (see Figure 13); the $\mathrm{S}^{1}-\mathrm{N}^{1}-\mathrm{P}^{1}$ bond angle widens by $2.8^{\circ}$ for $\mathrm{F}$ as a substituent on $\mathrm{S}$ and by $6.3^{\circ}$ for $\mathrm{Cl}$ as a substituent on $\mathrm{S}$. The $\mathrm{P}^{1}-\mathrm{N}^{2}-$ $\mathrm{P}^{2}$ bond angle of the "phosphazene end" of the monomer
Table 3. Torsional Barriers Obtained by Rigid Rotor and Relaxed Scans

\begin{tabular}{ccc}
\hline & \multicolumn{2}{c}{ torsional barrier $(\mathrm{kcal} / \mathrm{mol})$} \\
\cline { 2 - 3 } bond & relaxed scan & rigid rotor scan \\
\hline $\mathrm{N}^{1}-\mathrm{P}^{1}, \mathrm{X}=\mathrm{F}$ & 3.05 & 10.5 \\
$\mathrm{~N}^{1}-\mathrm{P}^{1}, \mathrm{X}=\mathrm{Cl}$ & 3.5 & 11.9 \\
$\mathrm{~S}^{1}=\mathrm{N}^{1}, \mathrm{X}=\mathrm{F}$ & 1.5 & 3.3 \\
$\mathrm{~S}^{1}=\mathrm{N}^{1}, \mathrm{X}=\mathrm{Cl}$ & 1.85 & 7.6 \\
$\mathrm{~N}^{2}-\mathrm{P}^{2}, \mathrm{X}=\mathrm{F}$ & 2.79 & 8.45
\end{tabular}

shows the most dramatic change during a rotation: it is widened by $25.5^{\circ}$ in the eclipsed position ( $\mathrm{F}$ as a substituent on S). The widening of the $\mathrm{S}-\mathrm{N}-\mathrm{P}$ and $\mathrm{P}-\mathrm{N}-\mathrm{P}$ bond angles decreases the repulsive interaction of the $\mathrm{S}(\mathrm{OX})$ and the $\mathrm{P}\left(\mathrm{Cl}_{2}\right)$ moieties and therefore lowers the torsional barriers. The torsional barriers for the three torsional motions are shown in Table 3.

The barriers range from 1.5 to $3.5 \mathrm{kcal} / \mathrm{mol}$. The difference between the torsional barriers which were obtained, on the one hand, by geometry-optimized molecular structures and, on the other hand, by the rigid rotor approximation shows that the flexibility of the $\mathrm{S}-\mathrm{N}-\mathrm{P}$ and $\mathrm{P}-\mathrm{N}-\mathrm{P}$ bond angles contributes significantly to the torsional mobility of the PTP backbone.

Effects of Electron Correlation. In order to estimate the effect of electron correlation on the torsional potentials, we carried out single-point 2nd-order MøllerPlesset perturbation theory (MP2) calculations. We used the RHF/6-31G* geometries of the stable geometry of mimic 2 and the geometry-optimized RHF/6-31G* structure of the eclipsed conformation for a rotation around the $\mathrm{P}^{1}-\mathrm{N}^{1}$ bond. In both cases we used the compounds with fluorine as a substituent $\mathrm{X}$ on sulfur. Electron correlation lowered the energies for the stable structure and the eclipsed conformation $(E$ RHF) $=$ -3333.6997 hartree, $E(\mathrm{RMP} 2)=-3335.6841$ hartree for the stable conformation; $E(\mathrm{RHF})=-3333.6948$ hartree, $E(\mathrm{RMP} 2)=-3335.6794$ hartree for the eclipsed conformation). The difference between the energies of 
stable conformation and eclipsed conformation drops from 3.05 to $2.96 \mathrm{kcal} / \mathrm{mol}$ (which is a $3 \%$ change) if electron correlation effects are considered. Thus, electron correlation effects have only a minor influence on the magnitude of the torsional barrier.

\section{Summary and Outlook}

We used ab initio molecular orbital calculations on short-chain model compounds in order to study the conformation, bonding, and chain flexibility of substituted poly(thionylphosphazenes) (PTPs) [(NSOX)$\left.\left(\mathrm{NPCl}_{2}\right)_{2}\right]_{n}(\mathrm{X}=\mathrm{F}$ or $\mathrm{Cl})$. The results of the geometry optimizations show that the model compounds adopt a nonplanar trans-cis geometry as their lowest energy conformation. The nonplanarity is caused by the two different substituents on sulfur. Therefore, it is likely that the PTP polymer will form a helix in its extended conformation. On the basis of the geometries of the short-chain analogues, we determined a $12 / 5$ helix for the polymers with chlorine as a substituent on phosphorus and fluorine or chlorine as a substituent on sulfur. We found a stable cis-trans conformation of mimic 2 as a higher energy conformation.

The torsional flexibility of five bonds of the main chain was investigated with rigid rotor scans and geometry optimizations of selected rotamers. A comparison of the results of the rigid rotor scans with the results for geometry-optimized rotamers showed that the flexibility of the $\mathrm{S}-\mathrm{N}-\mathrm{P}$ and $\mathrm{P}-\mathrm{N}-\mathrm{P}$ bond angles contributes significantly to the rotational flexibility of the polymer backbone. During a full rotation these angles are bent open by up to $25.5^{\circ}$. The torsional barriers range from 1.5 to $3.5 \mathrm{kcal} / \mathrm{mol}$. The change from chlorine to fluorine as a substituent on sulfur lowers the torsional barriers and widens the minima of the torsional potentials. This increases the flexibility of the polymer backbone and therefore is consistent with the lowering of the glass transition temperature $\left(T_{\mathrm{g}}(\mathrm{X}=\mathrm{F})=-56^{\circ} \mathrm{C}, T_{\mathrm{g}}(\mathrm{X}=\mathrm{Cl})\right.$ $=-46{ }^{\circ} \mathrm{C}$ ). Electron correlation has a negligible influence on the height of the torsional barrier. In addition, the results of the rigid rotor scans show that the torsional flexibility of a bond of the main chain is governed by its immediate molecular environment. This underlines the relevance of the calculations on the chain flexibility of low molecular weight analogues for the polymeric system.

The charge density distribution of the valence electrons of the model compounds can be described by the island delocalization model developed by Dewar. The results indicate strong charge separations along the skeleton of the model compounds. This suggests that electrostatic interactions will play an important role in intra- and intermolecular interactions.

Acknowledgment. The authors thank the Natural Science and Engineering Research Council of Canada (NSERC) and the Ontario Centre for Materials Research (OCMR) for financial support. We thank the reviewers of this paper for their constructive criticism. It is a pleasure to thank Ms. Anne Klemperer and Mr. Jason Massey for their help with the preparation of the manuscript.

\section{References and Notes}

(1) Mark, J. E.; Allcock, H. R.; West, R. Inorganic Polymers; Prentice-Hall: Englewood Cliffs, NJ, 1992.

(2) (a) Foucher, D. A.; Ziembinski, R.; Tang, B.; Macdonald, P. M.; Massey, J.; Jaeger, R.; Vancso, G. J.; Manners, I. Macromolecules 1993, 26, 2878. (b) Manners, I. Adv. Mater. 1994, 6, 68. (c) Sheats, J. E.; Carraher, C. E.; Pittman, C. U. Metal Containing Polymer Systems; Plenum: New York, 1985.

(3) (a) Allcock, H. R. Adv. Mater. 1994, 6, 106. (b) Allcock, H. R. J. Inorg. Organomet. Polym. 1992, 2, 197.

(4) Liang, M.; Manners, I. J. Am. Chem. Soc. 1991, 113, 4044.

(5) (a) Ni, Y.; Stammer, A.; Liang, M.; Massey, J.; Vancso, G. J.; Manners, I. Macromolecules 1992, 25, 7119. (b) Manners, I. Polym. News 1993, 18, 133.

(6) Gibbs, J. H.; Di Marzio, E. A. J. Chem. Phys. 1958, 28, 373.

(7) Sundararajan, P. R. Macromolecules 1990, 23, 2600.

(8) Sundararajan, P. R. Macromolecules 1993, 26, 344.

(9) Hehre, W. J.; Radom, L.; Schleyer, P. v. R.; Pople, J. A. Ab Initio Molecular Orbital Theory; John Wiley \& Sons: New York, 1986.

(10) Davidson, R. D.; Feller, D. Chem. Rev. 1986, 86, 681.

(11) Frisch, M. J.; Trucks, G. W.; Head-Gordon, M.; Gill, P. M. W.; Wong, M. W.; Foresman, J. B.; Johnson, B. G.; Schlegel, H. B.; Robb, M. A.; Replogle, E. S.; Gomperts, R.; Andres, J. L.; Raghavachari, K.; Binkley, J. S.; Gonzalez, C.; Martin, R. L.; Fox, D. J.; Defrees, D. J.; Baker, J.; Stewart, J. J. P.; Pople, J. A. Gaussian 92, Revision C, Gaussian, Inc., Pittsburgh PA, 1992.

(12) Glendening, E. D.; Reed, A. E.; Carpenter, J. E.; Weinhold, F. NBO Version 3.1 .

(13) CERIUS molecular modeling software for materials research from Molecular Simulations Inc. of Burlington, MA, and Cambridge, U.K.

(14) Portions of the work described in this paper have been briefly reported earlier in: Lagowski, J. B.; Jaeger, R.; Manners, I.; Vancso, G. J. Polym. Prepr. (Am. Chem. Soc., Div. Polym. Chem.) 1993, 34, 326.

(15) Lagowski, J. B.; Jaeger, R. Int. J. Quantum Chem., in press.

(16) Liang, M.; Lough, A. J.; Manners, I., unpublished results.

(17) Allcock, H. R.; Tollefson, N. M.; Arcus, R. A.; Whittle, R. R. J. Am. Chem. Soc. 1985, 107, 5166.

(18) Allcock, H. R.; Ngo, D. C.; Parvez, M.; Whittle, R. R.; Birdsall, W. J. J. Am. Chem. Soc. 1991, 113, 2628.

(19) Allcock, H. R.; Arcus, R. A.; Stroh, E. G. Macromolecules 1980, 13,919 .

(20) Hasselbring, R.; Roesky, H. W.; Rietzel, M.; Witt, M.; Noltemeyer, M. Phosphorus, Sulfur, Silicon Relat. Elem. 1992, 72, 209.

(21) Roy, A. K.; Burns, G. T.; Lie, G. C.; Grigoras, S. J. Am. Chem. Soc. 1993, 115, 2604

(22) Chatani, Y.; Yatsuyanagi, K. Macromolecules 1987, $20,1042$.

(23) Ferris, K. F.; Duke, C. B. Int. J. Quantum Chem., Quantum Chem. Symp. 1989, 23, 397

(24) Longuet-Higgins, H. C.; Salem, F. R. S.; Salem, L. Proc. R. Soc. London A 1959, 251, 172.

(25) Dewar, M. J. S.; Lucken, E. A. C.; Whitehead, M. A. J. Chem. Soc. 1960, 2423.

(26) Mulliken, R. S. J. Chem. Phys. 1955, 23, 1833, 1841, 2338, 2343.

(27) Reed, A. E.; Weinstock, R. B.; Weinhold, F. J. Chem. Phys. $1985,83,735$.

(28) Massey, J.; Jaeger, R.; Edwards, M.; Gates, D.; Manners, I.; Vancso, G. J., submitted to Macromolecules.

MA941082C 